Théologiques

Théologiques

\title{
Qui a peur de la communauté?
}

\section{Jacques T. Godbout}

Volume 6, numéro 1, mars 1998

Autres regards sur la laïcité

URI : https://id.erudit.org/iderudit/024953ar

DOI : https://doi.org/10.7202/024953ar

Aller au sommaire du numéro

\section{Éditeur(s)}

Faculté de théologie de l'Université de Montréal

\section{ISSN}

1188-7109 (imprimé)

1492-1413 (numérique)

Découvrir la revue

\section{Citer cet article}

Godbout, J. T. (1998). Qui a peur de la communauté? Théologiques, 6(1), 29-38. https://doi.org/10.7202/024953ar

\section{Résumé de l'article}

La laïcité est beaucoup associée à la mentalité et à la culture françaises. En établissant quelques différences majeures qui marquent les modèles juridiques français (démocratie directe) et anglo-saxon (démocratie représentative) l'auteur montre comment la méfiance des français pour la notion de communauté vient de l'absence d'une vision de la société existant par elle-même, indépendamment du pouvoir politique et de l'Etat. Cette utopie de la communauté à l'échelle des grands nombres exclus les communautés " concurrentes » et verse dans une hypertrophie de la Raison, oblitérant tout autre aspect du réel qui pourrait permettre d'organiser le social autrement. L'école laïque prétend former à la liberté de penser par la seule Raison.
Tous droits réservés @ Faculté de théologie de l'Université de Montréal, 1998
Ce document est protégé par la loi sur le droit d'auteur. L’utilisation des services d’Érudit (y compris la reproduction) est assujettie à sa politique d'utilisation que vous pouvez consulter en ligne.

https://apropos.erudit.org/fr/usagers/politique-dutilisation/ 


\title{
Qui a peur de la communauté?
}

\author{
Jacques T. GODBOUT \\ IRNS Urbanisation
}

\section{RÉSUMÉ}

La laïcité est beaucoup associée à la mentalité et à la culture françaises. En établissant quelques différences majeures qui marquent les modèles juridiques français (démocratie directe) et anglo-saxon (démocratie représentative) l'auteur montre comment la méfiance des français pour la notion de communauté vient de l'absence d'une vision de la société existant par elle-même, indépendamment du pouvoir politique et de l'État. Cette utopie de la communauté à l'échelle des grands nombres exclus les communautés " concurrentes " et verse dans une hypertrophie de la Raison, oblitérant tout autre aspect du réel qui pourrait permettre d'organiser le social autrement. L'école laïque prétend former à la liberté de penser par la seule Raison.

Laicity is often associated with the mentality and the culture of the French. In establishing some of the major differences that distinguish the French juridical model (direct democracy) from the Anglo-saxon model (representative democracy), the author shows how the mistrust of the notion of community by the French, arises from the lack of a vision of a society which exists by itself, independent from political power and from the State. This utopia of the community on a large scale excludes " rival * communities and falls into a hypertrophy of Reason, forgetting all other aspects of reality that could permit a different kind of social organization. The lay school claims this formation through the liberty of thinking by Reason alone.

\section{1. À propos de laïcité}

Dans une église les hommes doivent se découvrir (et les femmes se couvrir). Dans une mosquée et dans un temple bouddhiste, on se déchausse. Dans ce lieu sacré de la modernité à la française qu'est l'école laïque, on doit se dépouiller de ses liens sociaux, y pénétrer comme individu isolé, sans racine, sans histoire. À cette condition on aura accès à la lumière de la Raison. Mais il faut se présenter nu, rejeter le vieil homme comme dans les rites d'initiation que nous décrivent les ethnologues. 
Pourquoi ce renoncement est-il aussi nécessaire dans un contexte laïc, au point où on a vu la société tout entière se mobiliser parce qu'une adolescente a violé cette règle en 1989 , alors que la France fêtait le bicentenaire de sa révolution? Pourquoi, alors que dans la plupart des sociétés modernes, et particulièrement dans les démocraties anglo-saxonnes, une telle attitude semble-t-elle beaucoup moins présente? Au contraire souvent, dans ces sociétés, on semble fonder la modernité sur la tolérance de tels comportements. D'ailleurs même les sociétés archaïques ont rarement obligé le missionnaire à se dévêtir de sa soutane, - symbole religieux s'il en est - et à revêtir le pagne! La tolérance ne ferait-elle donc pas partie elle aussi des vertus de la modernité, même laïque? Il est bien connu par ailleurs que la France se méfie de la communauté, et tout particulièrement de toute reconstitution potentielle de communautés à l'intérieur de «sa" société. Elle a fait de la démocratie un moyen de la nécessaire destruction de la communauté. Pourquoi une telle méfiance qu'on ne semble pas retrouver dans les autres démocraties modernes?

C'est justement en revenant à ce moment fondateur qu'est la Révolution que l'on peut comprendre cette conception de la modernité. L'hypothèse que j'aimerais développer est la suivante. Cette attitude peut s'expliquer par le fait que la citoyenneté «à la française » se définit ellemême comme communauté, mais sans le dire (une véritable communauté est-elle jamais consciente d'elle-même?), ce qui la rend "concurrentielle " avec les autres communautés qui pourraient se former en son sein. Le modèle français de démocratie conserve l'utopie de la communauté à l'échelle des grands nombres. Concrètement cela signifie l'impossible projet de vouloir fonctionner dans une démocratie représentative comme si on était dans une démocratie directe. La France révolutionnaire a projeté le modèle de la démocratie directe, propre aux petites sociétés, sur une grande société. La démocratie directe est " cet instrument qui sert à fabriquer de l'opinion unanime" (FURET 1978 1 ). Or un système de démocratie représentative ne peut fonctionner au contraire que sur la reconnaissance de l'impossibilité d'atteindre cette unanimité, ce qui entraîne l'institutionnalisation de l'opposition et du pluralisme. Cette utopie communautaire appliquée à toute une société, c'est la quadrature du cercle du modèle français de démocratie, et c'est ce qui le distingue du modèle anglo-saxon.

1 Pour plus de détails sur la différence entre démocratie directe et démocratie représentative, voir Godbout, 1987, 1990 


\section{Deux modèles}

Explicitons ces propositions en rappelant d'abord certains traits des conceptions française et anglo-saxonne des droits de l'homme et de la démocratie. Historiquement autant que conceptuellement, le modèle anglo-saxon est fondé sur le droit à l'opposition, sur la nécessité de disposer des moyens de se débarrasser d'un mauvais gouvernement. Ultimement ce droit repose sur la tradition. Cela ne signifie pas une attitude d'obéissance aveugle et non raisonnée, comme on a tendance à le concevoir à partir du modèle français. Hayek a bien illustré ce point. «Nous ne prétendons nullement que toute tradition soit, comme telle, sacrée et intangible pour la critique; nous disons simplement que la base de la critique de n'importe quel produit de la tradition doit toujours être cherchée dans d'autres produits de la tradition " (cité dảns Dupuy, 1992, p. 40). C'est la notion, fondamentale, du common law. Pour illustrer ces idées, rappelons qu'en Angleterre, le bill of rights a eu d'abord pour but de lutter contre l'arbitraire du pouvoir royal. On avait dressé une liste d'abus du pouvoir contre les pratiques individuelles et corporatives. En 1640, contre l'absolutisme du roi, on a fait appel à une légitimité ancienne pervertie par le roi; on a fait appel à l'histoire saxonne, aux droits historiques, pas aux droits naturels ou innés. Pour les anglo-saxons il n'y a pas de droit naturel; ou plutôt le droit naturel lui-même est fondé sur la common law, qui donne le droit de changer un gouvernement abusif. Le common law est la base de tout, fondé sur les précédents, mais avec la possibilité de les modifier compte tenu de la situation nouvelle. Fondé sur un ensemble de cas particuliers. On verra au contraire que l'universalité française refuse le particulier et les particularités (ce sont des "particularismes"). Dans le droit anglais remedies preceed rights. Autrement dit on trouve une solution à un problème et on en fait une loi après. La procédure est la base de la loi. C'est le common law qui protège de la tyrannie.

Ultimement bien sûr le pouvoir appartient au peuple, mais dans une démocratie représentative il n'est pas exercé par le peuple lui-même, mais par ses représentants, ce qui instaure une rupture radicale avec le modèle de la démocratie directe, seul modèle complet de démocratie comme l'a bien montré Baechler. La conception anglo-saxonne reconnaît ce fait : dorénavant le système politique échappe en partie au pouvoir du peuple. Il lui est en partie extérieur. Tout en demeurant ultimement fondé sur la communauté et ses traditions, la communauté n'est plus le seul principe de fonctionnement du système politique. D'où la nécessité de disperser le pouvoir par le jeu de balance des pouvoirs. Et également l'élection du pouvoir exécutif lui-même, qui consacre la rupture avec le modèle de la démocratie directe. Dans cette conception, il y a une méfiance fondamen- 
tale vis-à-vis tout pouvoir, même celui issu du peuple. Car ce pouvoir ne relève pas entièrement du principe communautaire. C'est un thème constant de la démocratie anglo-saxonne depuis Thomas Paine, thème bien analysé par Popper (1945) sous l'appellation "société ouverte". Si bien que cette démocratie pourrait se définir d'abord comme un droit à l'opposition (BURNHAM 1962). Le point de départ, ce ne sont pas des principes abstraits, et ce n'est pas la recherche d'un ordre nouveau. C'est d'abord négatif : comment se débarrasser d'un gouvernement mauvais?

Rien de tel l'autre côté de la Manche. La France, au moment de la Révolution, entend au contraire fonder un ordre nouveau, et un pouvoir nouveau. Elle se préoccupe moins des limites nécessaires du pouvoir, de tout pouvoir, une fois qu'il a échappé à la démocratie directe, et cherche un système ayant les mêmes propriétés que la démocratie directe, mais qui serait applicable aux grandes sociétés, qui serait universel et constituerait une sorte de garantie que la société possède un «bon " gouvernement. Dans ce projet rien n'est extérieur au système qu'on entend mettre en place. Le pouvoir trouve ses limites à l'intérieur même du pouvoir public, à l'intérieur de lui-même, dans les lois universelles qu'il a lui-même promulguées. C'est l'article 6 de la déclaration des droits de l'Homme, et c'est la notion d'intérêt général. La Révolution se méfie certes elle aussi du pouvoir; mais cette inquiétude vient en second par rapport à la volonté d'établir un ordre nouveau et universel. La révolution française manifeste "l'exigence d'une refondation radicale en raison ${ }^{2}$ ". Les moyens de se défendre contre ce pouvoir qui peut devenir abusif sont situés à l'intérieur même de cet ordre politique, ultimement dans la Constitution, et non à l'extérieur comme pour le common law, qui, de ce fait, fournit une base sociale au système politique. C'est fondamental.

Et c'est ce qui rend le modèle français de démocratie incapable de fonder la société sur les liens communautaires. Ces derniers au contraire se transforment en menace potentielle permanente pour l'État de droit, universel, laïc. Le modèle ne reconnaît rien d'autre que des citoyens abstraits, dépouillés de leurs caractéristiques propres et d'abord de leur liens, de leur réseaux sociaux. L'absence de référence au common law élimine la possibilité pour la société de détenir une légitimité en dehors de la construction abstraite des droits de citoyens isolés, seuls devant le système auquel ils doivent s'identifier pour simuler le modèle sous-jacent de l'unanimité consensuelle de la démocratie directe où tous décident et où le gouvernement n'a donc pas d'importance, puisqu'il n'est tout au plus qu'un

2 Marcel GAUCHET, "Rabaud Saint-Etienne à la sortie du Désert », Le Monde de la révolution française, 11,1989, p. 20. 
exécuteur des décisions, au sens strict. Mais ce consensus réel, auquel on arrive dans de petites sociétés, est évidemment impossible à atteindre réellement dans une société comme la France. Il doit donc être remplacé par une notion abstraite, celle d'intérêt général que les groupes tenteront de s'approprier et d'interpréter. La méfiance naturelle des Français pour la notion de communauté vient de là, de l'absence d'une vision de la société existant par elle-même, indépendamment du pouvoir politique et de l'État. Tout lien communautaire se transforme alors en particularisme menaçant l'intérêt général et symbolisant un retour à l'ordre d'ancien régime. N'oublions pas que la France a pendant presque tout le XIXe siècle interdit l'association, ne pouvant pas imaginer l'existence d'intermédiaires entre l'État et chaque citoyen pris individuellement. On n'arrive pas à réaliser que l'intérêt général est second, qu'il est une émanation de ces liens concrets qui sont le roc de toute société, comme dit Marcel Mauss.

Le droit à l'opposition n'est pas premier; ce qui est premier c'est le bon gouvernement, fruit nécessaire du consensus de citoyens éclairés. C'est pourquoi on peut penser que la démocratie anglo-saxonne va jusqu'au bout de la logique de la démocratie représentative, alors que la démocratie française n'a jamais fait le saut et a toujours tendance à nier les conséquences de l'introduction de la représentation dans le système politique au profit d'une utopie de l'intérêt général. Historiquement ce problème, on le sait, s'est cristallisé autour du long débat sur le "mandat impératif " des députés. Or cette conception est une pure projection du modèle de la démocratie directe avec droit de veto suspensif. L'analyse de ce débat montrerait que l'on a jamais vraiment intégré la notion de représentation. Encore aujourd'hui des spécialistes de la théorie démocratique aussi importants que Baechler et Gauchet rejettent cette idée. Gauchet: « l'utopie de la représentation sur laquelle achoppera l'expérience politique de la Révolution." (1989, p. 20). Il cite Rabaud St-Étienne : "ce ne sont pas réellement les représentants qui font la loi, c'est le peuple, dont les représentants ne sont que l'organe". "Les mandataires représentent tout et ne se substituent en rien. "Il nous semble que contrairement à ce qu'affirme Gauchet, ce n'est pas l'utopie de la représentation, mais bien plutôt l'utopie de l'absence de la représentation, la négation de la réalité complexe de la représentation sous la fiction, comme si le peuple luimême décidait, -autrement dit l'utopie de la démocratie directe appliquée aux grands nombres-, qui est la source des totalitarismes modernes (et d'abord du communisme, cette utopie de la communauté des producteurs). La non reconnaissance de la réalité de la représentation comme distance entre le représentant et le citoyen est un problème de base de la démocratie moderne. Le degré de reconnaissance de la représentation et le statut qu'on lui accorde permettrait de situer les différentes démocraties représentatives sur un continuum, les plus tolérantes et les plus modestes 
étant celles qui sont les plus conscientes de leur origine, de leur base, celles qui savent que la démocratie représentative est la moins mauvaise approximation de la démocratie lorsqu'on se situe dans de grandes (en nombre...) sociétés, et que son dynamisme reposera toujours sur la vie démocratique des communautés qui la composent, avec les risques que cela entraîne, évidemment, contrairement à celles qui croient à la possibilité de fonctionner de la même façon qu'en démocratie directe, celles qui croient à une communauté des grands nombres qui serait de même nature que l'autre.

\section{Le méta-niveau inviolable}

Autrement dit la démocratie à la française arrive difficilement à faire ce que Hofsdadter (1980) appelle un «strange loop», une boucle étrange qui la ramène à son point de départ, à sa base, opération que réalise le common law en ramenant le pouvoir aux réseaux sociaux, en "rabaissant " ce complexe, orgueilleux et rationnel appareil politique moderne que sécrètent les démocraties représentatives à cette modeste base qu'est la coutume, la façon dont les ancêtres ont réglé le problème, non pas pour s'y conformer automatiquement comme on essaie toujours de nous le faire croire chez les partisans de la laïcité dans le but de valoriser la modernité, mais pour s'en inspirer et prendre une décision "éclairée " par autre chose que la raison abstraite. Tout se passe comme si la lä̈cité ne reconnaissait pas, dans toute société, "le fondement indisponible de la normativité ». (Ahmet Insel, MAUSS : 45). Je veux dire par là que toute société est ultimement fondée sur une adhésion à quelque chose, (même si c'est à la Raison...) auquel ses membres n'ont pas accès, et c'est le mythe de l'Occident de s'imaginer pouvoir y accéder par la raison ou autrement. Rappelant que depuis Gödel même la pensée mathématique reconnaît être soumise à la loi de l'incomplétude, Hofsdadter conclut que les boucles étranges et les méta-niveaux qui se multiplient théoriquement à l'infini sont toujours dépendants ultimement d'un méta-niveau qui ne peut pas être partie prenante, ne peut pas entrer dans la boucle, ce qu'il appelle le méta-niveau inviolable (HOFSTADTER 1980). L'Occident ne reconnaît plus l'existence d'un méta-niveau inviolable. Jusqu'à récemment il était représenté par le roi, détenant directement son pouvoir de Dieu, instance hors du système, et auteur du système lui-même. La démocratie a supprimé ce méta-niveau (mais pas entièrement, puisque la monarchie se maintient dans une proportion importante de démocraties et, qu'aux USA, il est écrit sur la monnaie : "In God we trust »...), et a fait du système de gouvernement des hommes une boucle étrange endogène en renvoyant la source et le fondement ultime du pouvoir des gouvernants aux gouvernés euxmêmes, c'est-à-dire à ceux qui devraient être situés, et qui le sont-au niveau le plus bas. Ils deviennent en même temps le méta-niveau: 
système invraisemblable, boucle totalement étrange, et très dérangeante pour les gouvernants. Il est alors bien normal que ces derniers aient la tentation constante de "corriger " ce paradoxe, de rationaliser, de trouver un méta-niveau plus " normal "..., surtout ceux qui ne sont pas choisis (élus) par ceux d'en bas et qui détiennent malgré tout du pouvoir. Et il est bien normal également de chercher ce méta-niveau dans les mythes actuels dominant la société. La Raison et la Science, par exemple. C'est pourquoi la laïcité entretient aujourd'hui des complicités avec la technocratie et constitue l'une des tentations permanentes de la démocratie, visant à identifier le méta-niveau au mythe dominant actuellement la société, origine et fondement absolue de la vérité : la Science. Si les décisions étaient fondées sur la science on mettrait fin à cette boucle gênante qui ramène tout à l'ignorant " homme de la rue ". L'idéologie technocratique est cette tentation permanente de la démocratie de se trouver un méta-niveau inviolable comme "dans le bon vieux temps", de remplacer le Roi et Dieu par la Science, de ne pas assumer son état "désenchanté ", comme dirait Weber. Et l'application aux systèmes de gouvernement de l'analyse systémique, fondée sur des modèles de niveaux hiérarchiques sans possibilité de boucle étrange, a le même résultat, en transformant les rapports gouvernants-gouvernés en rapports de feedback, c'est-à-dire où le rôle des gouvernés n'est plus que de "feeder ", "nourrir " les niveaux supérieurs. Le peuple est ainsi réduit au seul statut de niveau inférieur. Il est pertinent ici de rapporter la différence que fait Hofsdadter entre feedback et boucle étrange (ou tangled hierarchy et linear hierarchy), qui s'applique parfaitement au domaine politique (alors que Hofsdadter réfléchit sur l'intelligence artificielle). "A Tangled Hierachy occurs when what you presume are clean hierarchical levels take you by surprise and fold back in a hierarchy-violating way. (...) A simple tangle, like feedback, doesn't involve violations of presumed level distinctions. " (p. 691). On comprend pourquoi tout bureaucrate tend constamment à transformer ses rapports avec les citoyens en feedback. Sans cette boucle étrange qui ramène le sommet à la base, un État n'est qu'une bureaucratie, les liens sociaux se diluent dans la notion d'intérêt général et le citoyen se retrouve seul. Cette boucle étrange est indispensable à la démocratie représentative pour maintenir un référent (la société) à l'extérieur du système politique, et surtout pour ne pas le couper abstraitement de son passé et maintenir ainsi son aspect communautaire, cet ensemble de liens que devrait précisément abandonner (théoriquement) tout individu moderne entrant dans le système de démocratie représentative.

Et tout enfant pénétrant dans l'école laïque. Ce qui nous ramène à la question de départ : pour accéder à la citoyenneté française il faut abandonner toute appartenance communautaire, sauf en son for intérieur et dans les lieux (églises, etc.) prévus à cet effet. (Et on croit que de cette 
façon on évite les ghettos, contrairement au pluralisme anglo-saxon....). La laïcité, au départ, c'est simplement une entente, un compromis, un traité de paix entre les catholiques et les anti-religieux. Mais rapidement elle va devenir la conception française des rapports entre l'État et la religion, puis la société civile, conception qui a comme particularité de se considérer comme la seule qui permet d'accéder aux Lumières, à la vérité qu'impose la Raison. La laïcité implique que l'école soit soustraite à la société civile et les enfants à l'autorité des parents lorsqu'ils sont à l'école, car ils y viennent pour apprendre à devenir libres, à penser par euxmêmes, et à cette fin il est nécessaire qu'ils fassent table rase de leurs croyances, quitte à les retrouver plus tard, librement. Ils doivent d'abord acquérir le savoir encyclopédique qui s'impose par lui-même, par sa seule force, celle de la raison. L'élève devient libre après avoir été exposé à cette vérité universelle qui s'impose à lui. Mais dans un premier temps il doit abandonner ses croyances actuelles, écarter ce qui fait obstacle à la libération de la pensée et à l'autorité de la raison. Il y a là une foi dans les vérités universelles qui constitue un méta-niveau qui empêche la démocratie de faire sa boucle étrange et de revenir au peuple. Il y a une métaréférence, il y a la raison comme mythe. Pris au pied de la lettre, cela signifierait que tous les Français qui vont dans les écoles privées religieuses n'accèdent pas à cette vérité si l'école laïque en est une condition nécessaire; ce qui signifierait aussi évidemment que les citoyens des autres pays sont moins libres que les Français, que ceux des USA par exemple, qui ont une autre conception de la démocratie, ne sont pas émancipés, ce qui les discrédite a priori dans leur prétention à discuter avec un Français de la vérité, n'ayant pas été formés à la découverte de la vérité qui s'impose à la raison.

Pourquoi une telle vision de l'école est-elle un mythe? Voyons pratiquement ce que l'élève apprend. Certes que $2+2$ font 4 , vérité qui tend effectivement à devenir universelle (dans la base $10 \ldots$ ). Mais cela on l'apprend partout, que l'école soit laïque ou non, française ou non. Ce qu'il apprend de spécifique, ce sont les valeurs de la société française, son histoire, sa géographie. (Quelqu'un a-t-il déjà démontré que l'histoire de France enseignée dans les écoles laïques était plus "objective " que l'histoire enseignée dans les écoles des pays n'adhérant pas à la doctrine de la laïcité?) C'est aussi cette valeur à l'effet que sans école laiqque, les individus ne peuvent pas devenir libres, s'émanciper, accéder à cette vérité qui s'impose d'elle-même. C'est de toute évidence un ensemble de vérités discutables. On peut d'ailleurs se demander pourquoi il y a autant de débats sur la laïcité chez les intellectuels Français eux-mêmes, qui ont pourtant tous reçu cette formation, la seule qui donne accès à la liberté et à la vérité, et que si cette vérité s'impose à la raison, ils devraient tous en penser la même chose, au moins en ce qui concerne cette vérité de base du 
système (cette méta-vérité...) à l'effet que c'est le moyen d'accéder aux vérités universelles. (Sans doute cela s'explique-t-il par le fait que les futurs petits intellectuels français fréquentent beaucoup trop l'école privée...) Cette vérité qui s'impose à la raison devrait entraîner le consensus. Matalon: " une preuve correcte s'impose par elle-même, au moins aux personnes compétentes et sans préjugés, et donc elle doit normalement entraîner le consensus" (1986, p. 270). Ceux qui ne sont pas d'accord avec cette conception de la laïcité sont donc soit incompétents, soit préjugés. Cette vision d'une vérité qui s'impose rationnellement à nous entraîne automatiquement une dévalorisation a priori de l'interlocuteur, comme le font souvent les religions, surtout monothéistes. On retrouve cette idée du consensus propre à la démocratie directe, mais utopie dangereuse dans la démocratie représentative.

Le modèle français de démocratie conduira-t-il à une solution originale aux problèmes actuels? Certes ses défenseurs peuvent se consoler en constatant que les autres modèles ne sont pas dans une situation beaucoup plus avantageuse aujourd'hui. L'immigration pose un défi à toutes les nations occidentales. Mais ne pourrait-on pas penser que communaurés et État peuvent se soutenir, devenir complémentaires pour affronter les problèmes actuels, au lieu de se nier mutuellement. Il est significatif que le bicentenaire de la révolution française, il y a quelques années, n'ait pas donné lieu à un débat de fond sur ce thème, sur cette double conception de la société moderne. Et ce n'est pas sans ironie qu'on peut constater que ce débat s'est finalement produit "grâce " à des écolières et à leurs foulards. Peut-être contribuera-t-il à diminuer l'attachement de la France à cette conception abstraite d'une citoyenneté communautaire, cet intégrisme de la modernité qui consiste à affirmer qu'il n'y a qu'une seule façon d'être moderne, la nôtre.

\section{Bibliographie}

BAECHLER, Jean. 1985. Démocraties. Paris, Calmann-Levy.

BURNHAM, James. 1962. The Managerial Revolution. New York, Penguin Books, (première édition 1942.) Version française : 1969. L'ère des organisateurs. Paris, Calmann-Lévy.

FURET, François. 1978. Penser la Révolution française. Paris, Gallimard (Folio).

GAUCHET, Marcel. 1989. "Rabaud Saint-Étienne à la sortie du Désert ", Le Monde de la révolution française, 11.

GODBOUT, Jacques T. 1987. La démocratie des usagers. Montréal, Boréal. 
GODBOUT, Jacques T. 1990. "Démocratie directe et démocratie représentative: à propos de Démocraties de Jean Baechler ", Revue du M.A.U.S.S., $7: 15-28$.

HOFSTADTER, Douglas R. 1980. Gödel, Escher, Bach : an Eternal Golden Braid. New York, Vintage Books. Titre français : 1985. Godel, Escher, Bach : les brins d'une guirlande éternelle, Paris, InterÉditions.

MATALON, Benjamin. 1986. "Sociologie de la science et relativisme", Revue de synthèse, $4: 267-290$.

POPPER, Karl R. 1945. The Open Society and Its Enemies. Londres Buttler and Tamer. Version française : 1979. La société ouverte et ses ennemis, Paris, Seuil, Tome 1:L'ascendant de Platon; tome 2 : Hegel et Marx.

TOCQUEVILLE, Alexis de. (1968, c1835-1840). De la démocratie en Amérique. Paris, Gallimard. 